\title{
Covert bronchogenic cyst as a cause of life-threatening cardiopulmonary impairment
}

\author{
EA Mather, JIC Hogg, ARO Miller
}

\begin{abstract}
Summary
We present a case in which a covert bronchogenic cyst resulted in two hospital admissions within three years, the second of which was characterised by lifethreatening cardiopulmonary compromise requiring sustained support on the intensive therapy unit.
\end{abstract}

Keywords: bronchogenic cyst, cardiopulmonary impairment

\section{Case history}

Three years ago, this 50-year-old woman had been admitted to another hospital with a cardiac dysrhythmia and a large pericardial effusion requiring drainage. Following drainage, a chest radiograph showed cardiomegaly and left atrial enlargement. Echocardiography suggested the possibility of cor triatrium but, at subsequent transoesophageal echocardiography the left atrium was reported to be normal. It was felt that the problem had been precipitated by infection although no definitive evidence of this was ever obtained. During the next three years she remained entirely well and was back to full activity including tennis playing and vigorous walking.

This admission was preceded by four days of influenza-like symptoms and then precipitated by a six-hour episode of irregular palpitations with sweating and light-headedness but no chest pain. On admission, electrolytes were normal as was a cardiac enzyme series. Shortly after admission she had a period of fast atrial fibrillation which reverted spontaneously to sinus rhythm. Echocardiogram showed a very small pericardial effusion and a normal left atrium. Approximately 24 hours after admission the patient developed a cough, fever, dyspnoea, together with clinical and radiological features of a left lower lobe pneumonia. Oxygen and intravenous cefuroxime were started but within several hours the situation had worsened with the development of widespread pulmonary oedema requiring intravenous diuretics. Despite this treatment her respiratory failure progressed rapidly and she required admission to the intensive therapy unit and urgent intubation and ventilation. Inititally her condition deteriorated further and she required inotropic support but within 24 hours she had stabilised. She was extubated but still needed respiratory support for several days and she remained in intensive care for a total of nine days. Inotropes were withdrawn and captopril started. A pulmonary artery catheter never showed an elevated pulmonary capillary wedge pressure.

The rapid improvement of her clinical condition with ventilation suggested that this was primarily pulmonary oedema rather than pneumonia but no clear cause was found for the oedema. The only positive microbiological data was a significant rise in her titre of antibody to influenza $A$ virus. Therefore when she was off respiratory support she had a further transoesophageal echocardiogram done at another hospital. This confirmed a normal left atrium but suggested that there may be an external mass pressing on it. Left ventricle and cardiac valves were normal. Shortly after this investigation she was well enough for all medication to be withdrawn. She was discharged home and a subsequent computed tomography (CT) scan as an out-patient revealed a substantial middle mediastinal mass. The patient has since undergone thoracotomy and bronchogenic cyst excision.

\section{RADIOLOGY}

Serial chest radiographs taken at presentation, during admission to the intensive therapy unit and towards recovery consistently demonstrated appearances which were difficult to relate to the clinical picture and presumptive diagnosis. Widening of the carina and apparent enlargement of the left atrium were noted (figure 1). However, without supporting evidence of cardio-mediastinal pathology, it was assumed that the appearances were artefactual. The rapid clearance of the pulmonary consolidation did not seem compatible with a diagnosis of pneumonia and was more suggestive of pulmonary oedema (presumably caused by compression of pulmonary veins by the cyst). The abnormal mediastinal contour alone should have demanded further imaging at an early stage. However, this was delayed until prompted by a trans-oesophageal echocardiogram which supported the suggestion of a mediastinal mass.

Following the patient's discharge to convalesce at home a dynamic CT scan of the thorax was carried out as an out-patient. This confirmed a substantial middle mediastinal mass (figures 2 and 3 ). We emphasise the radiological lesson that a splayed carina and apparent left atrial enlargement can never be 


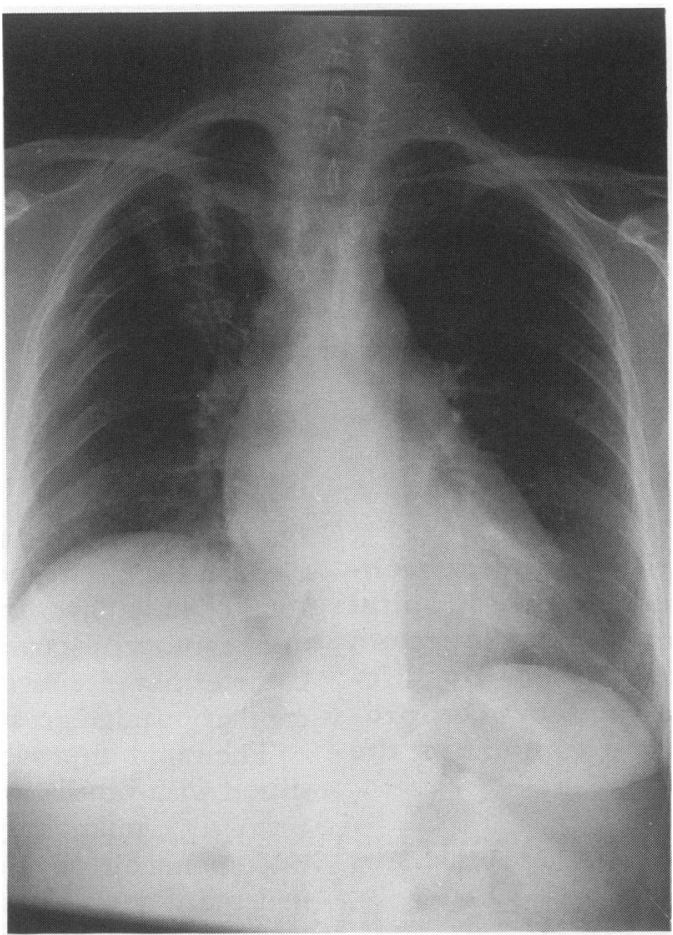

Figure 1 Chest X-rays towards recovery (8 April 94) showing widened carina, cardiomegaly, left atrial enlargement and residual linear right upper lobe shadowing

allowed to remain unexplained nor be lightly dismissed on repeated chest X-rays.

\section{Discussion}

The majority of bronchogenic cysts are a chance finding at chest radiography. ${ }^{1}$ Others may present as a result of inflammation or, less commonly, of haemorrhage within the cyst, or as a result of pressure upon the airways or oesophagus. ${ }^{2,3}$

Bronchogenic cysts are thought to result from abnormal budding of the tracheal primordium or abnormal branching of the tracheobronchial tree and are usually solitary ${ }^{3} ; 51 \%$ occur around the carina and some are attached to it although only $5 \%$ communicate with an airway. They are usually smooth, convex or round masses of homogenous density. ${ }^{4} \mathrm{Ex}$ tremes of size vary between cysts which are overlooked, to those occupying half the thorax with an average size of $3.6 \mathrm{~cm} .^{2}$ A 20 -year study of bronchogenic cyst cases reported that $27 \%$ present asymptomatically. Presenting symptoms are detailed in box $1 .^{5}$ Symptoms are commoner in those who have a communication with the airway. ${ }^{6}$

In the case presented it can be assumed that two hospitalisations were a result of inflammation within this covert bronchogenic cyst leading to swelling and pressure on the pulmonary veins. At the time of her CT scan, when the clinical picture had much improved, the images still demonstrate significant compromise of bronchial divisions and of some pulmonary veins. Consequently, the chest Xray appearances during her admission are
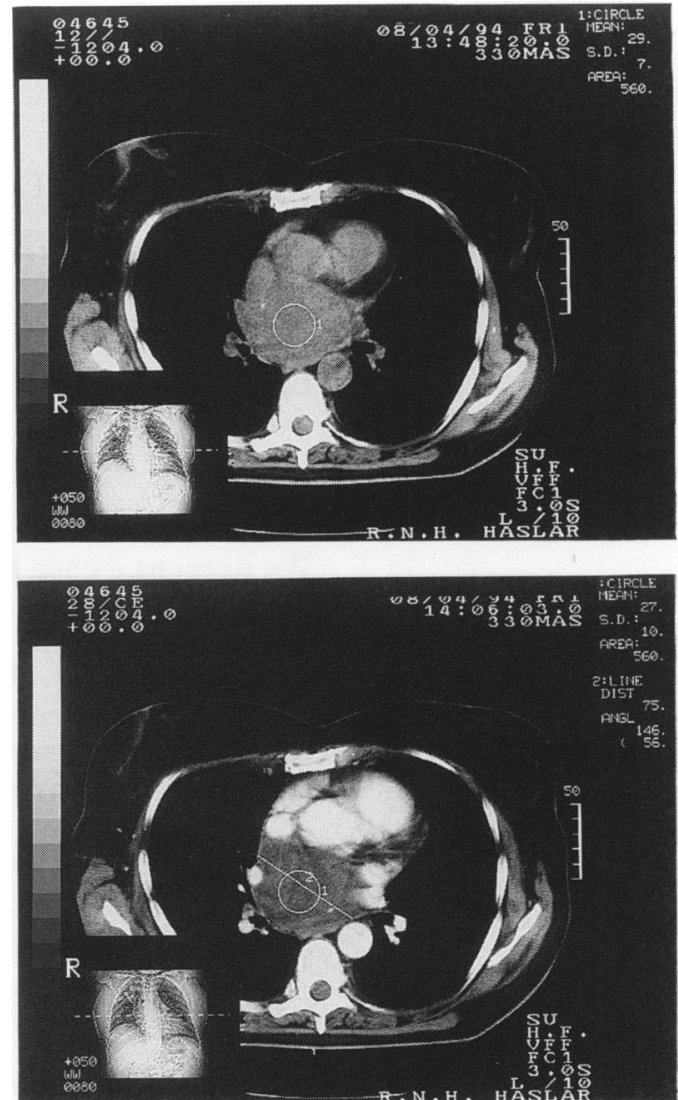

Figures 2 (upper) and 3 Pre and post contrastenhanced CT scans demonstrate a non-enhancing $7.5 \mathrm{~cm}$ subcarinal mass of attenuation value (29) consistent with viscous fluid and having intimate association with pulmonary arteries and veins and with the posterior aspect of the left atrium

Presenting symptoms of bronchogenic cysts

- cough

- dyspnoea

- pain

- fever

- pneumonia

- upper respiratory tract infection

- dysphagia

Box 1

\section{Learning points}

- splayed tracheal carina and apparent left atrial enlargement on chest radiograph should never be left unexplained

- CT scanning of the chest and trans-oesophageal echocardiography may be the best imaging methods to investigate this

- bronchogenic cysts can be difficult to diagnose and may require several different imaging techniques in order to identify them

Box 2

explained in retrospect as those of pressure effects upon bronchi and veins. Less well established is an association between bronchogenic cysts and cardiac dysrhythmia, which was amongst the presenting features, but the CT scans show pronounced mass effect upon 
the posterior aspect of the left atrium, and it is proposed that this may have adversely affected atrio-ventricular conduction.

Bronchogenic cyst diagnosis is often made by a plain chest radiograph. Ultrasonography is useful to establish the cystic nature of a mass but CT scanning is superior, affording a

1 Rogers LF, Osmer JC. Bronchogenic cysts: a review of 46 cases. AfR 1964; 91: 273-83.

2 Salyer DC, Salyer WR, Eggleston JC. Benign developmental cysts of the mediastinum. Arch Pathol Lab Med 1977; tal cysts of $136-9$.

3 101: 136-9. Surg 1948; 127: 476 .

Surg 1948; 127: 476.
4 Holesh S. Mediastinal tumours. In: Shanks SC, Kerley PHK, eds $A$ textbook of $X$-ray diagnosis by British authors. 4th edn. London: Lewis, 1973. reasonably confident pre-operative diagnosis of type 1 or 2 cystic adenomatous malformations. Type 3 lesions, being more solid, are less confidently diagnosed before surgery. Magnetic resonance imaging is useful to define intraspinal involvement in association with a mediastinal mass. ${ }^{7}$

5 DiLorenzo M, Collin PPC, Vaillancourt R, Duranceau A Bronchogenic cysts. $\mathcal{F}$ Pediatr Surg 1989; 24: 988-91.

6 Reed JC, Sobonya RE. Morphological analysis of foregut cysts in the thorax. $A \mathscr{F} R$ 1973; 120: 851-60.

7 Haddon MJ, Bowen A. Bronchopulmonary and
7 A. 7 Haddon $M J$, Bowen $A$. Bronchopulmonary and nosis and management. Radiol Clin N Am 1991;29: 241-54.

\title{
Surgical cure of hypertension in a patient with brainstem capillary haemangioblastoma containing neuropeptide $\mathrm{Y}$
}

\author{
SA Hedderwick, AE Bishop, AJ Strong, JM Ritter
}

\begin{abstract}
Summary
We describe a 29-year-old woman with persistent arterial hypertension which resolved following complete excision of a capillary haemangioblastoma from within the spinal cord at the cervicomedullary junction. Immunohistochemical staining was positive for neuropeptide $Y$ in capillary walls and nerves in the tumour. This raises the possibility of an association between neuropeptide $Y$ and the central control of blood pressure in man.
\end{abstract}

Keywords: hypertension, neuropeptide Y, haemangioblastoma, surgery

macology,

UMDS, Medical

School Building, Guy's

Hospital, London SE1

9RT, UK

SA Hedderwick

JM Ritter

\section{Department of}

Histochemistry, Royal

Postgraduate Medical

School, Hammersmith

Hospital, London

W12 ONN, UK

AE Bishop

\section{Department of}

Neurosurgery, The

Maudsley Hospital,

London SE5 8AZ, UK

AJ Strong

Correspondence to

Professor JM Ritter

Accepted 9 December 1994
A 29-year-old Jamaican woman presented to casualty after a head injury, complaining of right facial numbness and weakness, intermittent slurred speech and drooling of saliva. There was a three-month history of sweaty episodes which were not associated with anxiety or palpitations. Arterial blood pressure was $200 / 105 \mathrm{mmHg}$ sitting. There was no previous history of hypertension with the exception of a single reading of $139 / 100 \mathrm{mmHg}$ in her first and only pregnancy two years earlier. Her mother had been diagnosed as having essential hypertension aged 54 . On examination she had pyramidal weakness and loss of sensation in the right arm with abnormal sensation over the right side of her face. Supine hypertension (130-200/100-126 mmHg) with a marked postural drop in blood pressure (of up to $80 \mathrm{mmHg}$ ) on standing but with no change in pulse rate, was a consistent finding during subsequent hospital admission. The remainder of the examination was normal. Initial investigations revealed a neutrophil leucocytosis (total white cell count $16.2 \times 10^{9} / 1$ ) and a urinary tract infection due to Proteus spp. The following were normal or negative: serum creatinine and electrolytes, liver function, glucose, syphilis serology, urinary vanilyl mandelic acid to creatinine ratio on two occasions, renal ultrasound. Cerebrospinal fluid examination was acellular with a raised protein $(1383 \mathrm{mg} / 1$, normal range $<450 \mathrm{mg} / \mathrm{l})$. Autonomic function tests were normal with the exception of supine hypertension and postural blood pressure fall without a significant pulse rise. Magnetic resonance imaging (MRI) showed an enhancing lesion adjacent to the posterior arch of $\mathrm{Cl}$ extending down to the 